\title{
Spotlight on measles 2010: An ongoing outbreak of measles in an unvaccinated population in Granada, Spain, October to November 2010
}

\author{
B López Hernández ${ }^{1}$, J Laguna Sorinas², I Marín Rodríguez², V Gallardo García ${ }^{3}$, E Pérez Morilla³, J M Mayoral Cortés \\ (josem.mayoral.sspa@juntadeandalucia.es) ${ }^{3}$ \\ 1. Andalusian Health Service, Granada, Spain \\ 2. Provincial Health Office, Regional Ministry of Health, Granada, Spain \\ 3. Regional Ministry of Health of the Government of Andalusia, Seville, Spain
}

Citation style for this article:

López Hernández B, Laguna Sorinas J, Marín Rodríguez I, Gallardo García V, Pérez Morilla E, Mayoral Cortés JM. Spotlight on measles 2010: An ongoing outbreak of measles in an unvaccinated population in Granada, Spain, October to November 2010. Euro Surveill. 2010;15(50):pii=19746. Available online: http://www.

eurosurveillance.org/ViewArticle.aspx?Articleld=19746

Article published on 16 December 2010

On 13 October 2010, the Andalusian Epidemiological Surveillance Network was notified of one case of measles. By 15 November 2010, 25 confirmed cases of measles had been reported from Granada, southern Spain, of whom 22 were unvaccinated children under the age of 15 years. This ongoing outbreak involved a subpopulation with low vaccination coverage and parents with ideological objections to vaccination. As of 7 December the number of cases has reached 59.

\section{Background}

In Andalusia, Spain, a Plan of Action for Measles Elimination was approved in 2001 [1], following the recommendations of the World Health Organization (WHO) [2]. This Plan was created with the objective of eliminating indigenous measles by the year 2005, but elimination has not yet been achieved. The two strategic goals of the Andalusian Plan were to enhance the epidemiological surveillance system to facilitate early detection of cases and transmission control, and to increase the vaccination coverage in children in order to improve population immunity.

The measles-mumps-rubella (MMR) vaccine was introduced in 1984 in the Andalusian vaccination calendar for children at 15 months of age. In year 1990, a second dose was included in the calendar for children at 11 years of age. The age of administration of the second dose was changed to six years in 1999, and to three years in 2004 [3]. These changes were made in order to adapt the levels of immunity against measles in different age cohorts to the WHO proposals regarding the elimination of indigenous measles in the European Region.

Since 2001, two important measles outbreaks have occurred in Andalusia: one in 2003 in Almeria (180 cases; unpublished data) and the other in 2008 in Algeciras (155 cases) [4]. Both outbreaks mainly affected unvaccinated young adults, although unvaccinated children under 16 months of age were subsequently also affected.

Here we present preliminary data until 15 November on an ongoing outbreak of measles in Granada, southern Spain, a city with a population of approximately 234,000 inhabitants.

\section{Outbreak description}

On 13 October 2010, a suspected case of measles [5] in a 13-year-old girl was notified, and confirmed by serology (IgM-positive) two days later. The second case, in a 13-month-old child form the same neighbourhood was reported on 19 October 2010 by the same health centre. Both cases had attended a wedding reception where they had been in contact with a girl from another region in Spain who was diagnosed with measles when she returned home. Until 15 November 2010, a total of 25 cases of measles were confirmed (Figure 1).

The age of the cases ranged from seven months to 38 years. Nine of the 25 cases were under one year of age and 14 cases were under three years of age. Only three cases were older than 15 years.

Of the 25 cases, 21 were living in the same neighbourhood in Granada. Of these 21, 19 cases were younger than 15 years, and the other two were 24 and 29 yearsold. Eight of these children were too young to attend any educational centres, while the remaining 11 were attending the following centres:

- A secondary school located outside the affected neighbourhood: one case (living in the affected neighbourhood), no secondary cases;

- Primary school A, located in the affected neighbourhood: six cases;

- Primary school B, located in the affected neighbourhood: one case, no secondary cases; 
- Day care centre A, located in the affected neighbourhood: two cases;

- Day care centre B, located in the affected neighbourhood: one case, no secondary cases.

Four cases were living in other neighbourhoods in Granada or in nearby towns and were infected through transmission in hospital. These cases were 38 years, 7 months, 13 months and 16 months of age.
All cases were treated at the same hospital, 14 as outpatients, and 11 as inpatients. Two cases were diagnosed with bronchiolitis and pneumonia, respectively. Only one case, a six-year-old, had been vaccinated previously with one dose of MMR. The remaining cases were unvaccinated. Nineteen cases were laboratoryconfirmed and six cases were confirmed by epidemiological link with a confirmed case. To date measles virus genotype B3 was identified in two cases. Genotyping of the other cases is ongoing.

\section{FIGURE 1}

Confirmed measles cases by day of onset of rash, Granada, Spain, October-November 2010 ( $\mathrm{n}=25$ as of 15 November)

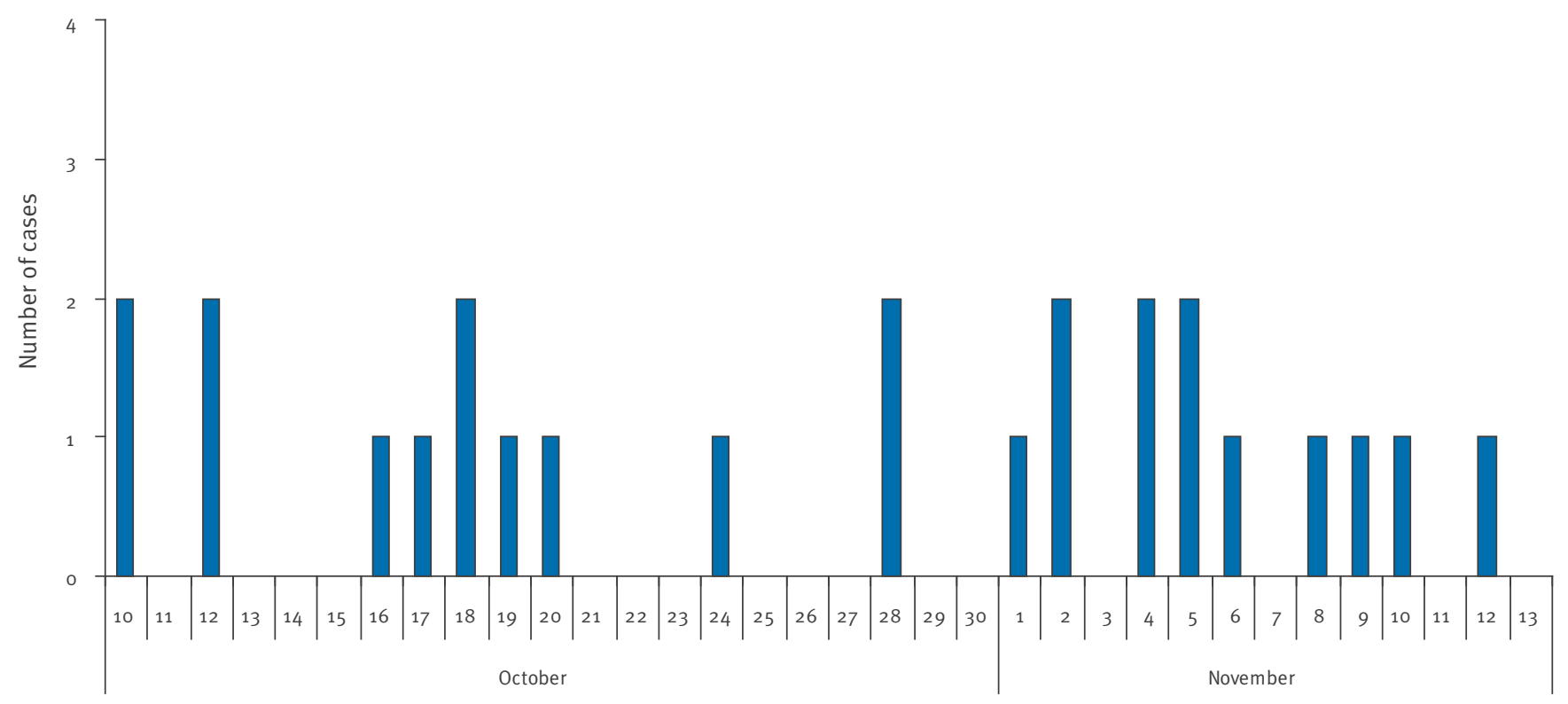

Date 2010

\section{FIGURE 2}

Confirmed measles cases by age, Granada, Spain, October-November 2010 ( $n=25$, as of 15 November)

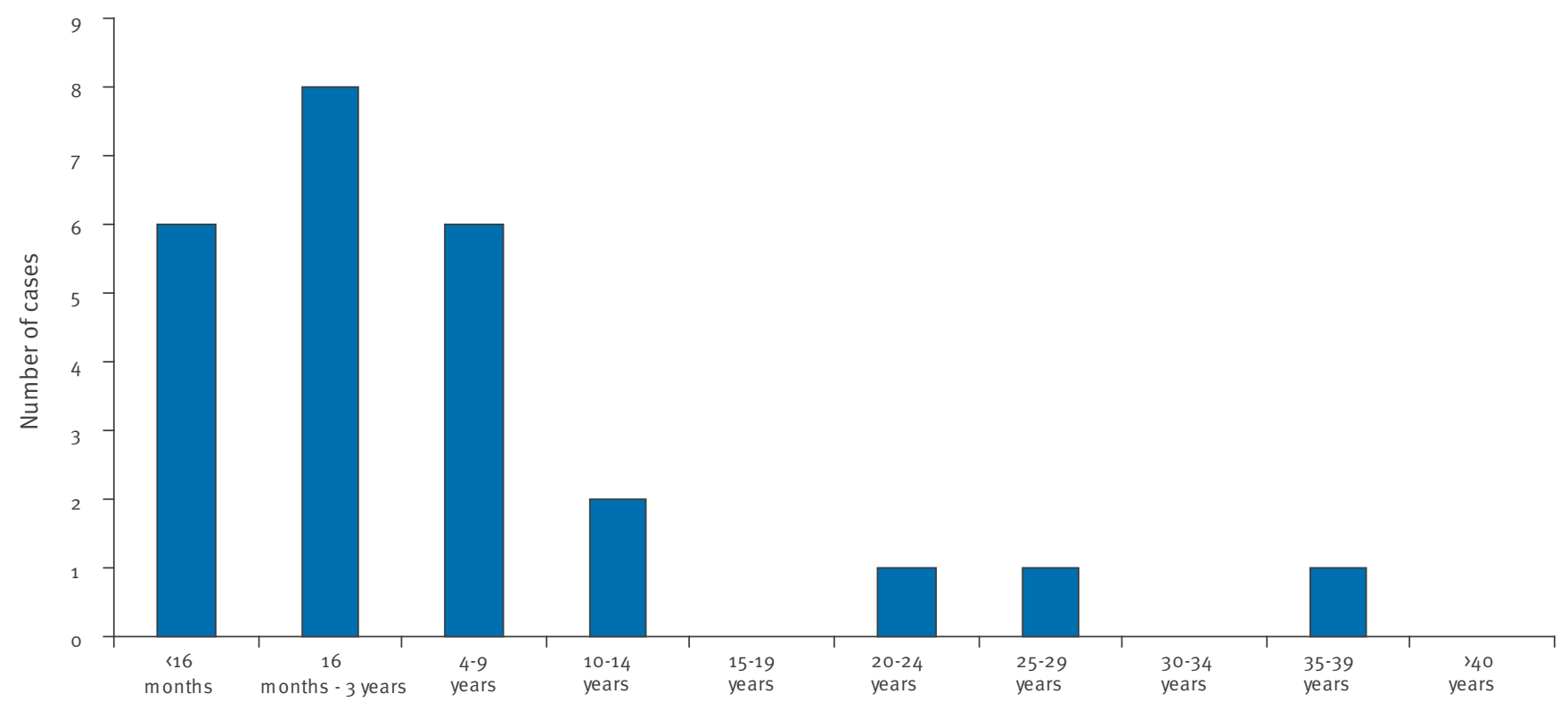

Age group 


\section{Control measures}

Control measures have been implemented in the four affected schools and the day care centre, in accordance with the Plan of Action for Measles Elimination and to Protocol of Alert of the Regional Ministry of Health [5]. The vaccination status of all children in the affected schools was reviewed and an MMR vaccine dose was offered to all children who were not fully vaccinated. Cases were excluded from school for at least four days after appearance of the exanthema. All affected school and day care staff younger than 40 years and without a history of the disease or documented evidence of vaccination were tested for susceptibility to measles and offered a dose of MMR vaccine.

In the affected day care centre, unvaccinated children aged between 12 and 15 months received one dose of MMR vaccine, and children aged between 6 and 11 months received one dose in the context of the current outbreak and are scheduled for a second dose at the age of 15 months according to the vaccination calendar. The unvaccinated contacts of cases or contacts with no history of measles were immunised with MMR vaccine in the first 72 hours after exposure, except for infants younger than six months, pregnant women and immunocompromised people, who were treated with antimeasles immunoglobulin.

It was recommended, until there are no more cases, to exclude from the affected centres individuals who were not vaccinated because of contraindications or another reason that excluded vaccination and who had no history of measles illness.

As a pre-exposure measure at the population level, a first dose of MMR vaccine is being administered to all children older than 11 months in the city of Granada and nearby towns that have reported one or more cases. A second dose of MMR vaccine will be administered to these children at three years of age according to the vaccination calendar. All health workers younger than 40 years working at healthcare centres in the outbreak area who had no history of measles or documented evidence of vaccination were vaccinated.

\section{Discussion}

There is an ongoing measles outbreak in Granada that began in a small community in the Albaycin neighbourhood who were not vaccinated due to ideological objections. The outbreak then spread to other unvaccinated people in the neighbourhood, mainly unvaccinated children under the age of 16 month. Outside this neighbourhood, secondary cases have to date only been detected in family contacts of the first cases (four cases) or people who had contact with the first cases in hospital (four cases). In 2010, similar outbreaks have been described in other European countries [6].

In the school with the highest number of cases (primary school $A$ ) and a low MMR vaccination coverage (about $60 \%$ ), the response to vaccination proposals was low at the beginning of the outbreak. We are currently working with parents of unvaccinated children in order to increase the response to vaccination, since many of these parents have no firm position against vaccination and there is a possible change of attitude. With these interventions vaccination coverage with one dose in this school has been increased to $95 \%$.

It is important to emphasise the hospital transmission in four cases admitted to the same hospital at the beginning of the outbreak, although there has not been any case among healthcare workers so far. To avoid transmission in waiting rooms, emergency services and inpatients in health centres of Granada, training sessions for the staff were organised reinforcing the preventive aspects.

As of 7 December 2010, a total of 59 confirmed cases of measles have been reported from Granada. Most of the cases were very small children or schoolchildren under the age of 15 years $(n=46)$. Few cases in young adults have been detected, in contrast to measles outbreaks in Algeciras (2008) [4] and Almeria (2003) (unpublished data). However, although coverage with MMR vaccine in Andalusia overall is appropriate to interrupt transmission of the disease in the population (above 95\%), a seroprevalence survey done in Spain and Andalusia in 1996 in the population between two and 40 years of age shows that there are more than $5 \%$ of susceptibles in the age cohorts born between 1997 and 1986 (currently between 24 and 33 years of age) [7]. Catch-up vaccination of these age groups has not been considered until now, so it is possible that the number of cases in this age group will further increase.

\section{Acknowledgements}

We would like to thank everyone who contributed to the outbreak study, with special mention to professionals at Granada and Metropolitan of Granada Health Districts, Provincial Health Office of Granada and Virgen de las Nieves Hospital Laboratory.

\section{References}

1. Plan de Acción para la Eliminación del Sarampión en Andalucía [Action plan for measles elimination in Andalusia]. Sevilla; Dirección General de Salud Pública y Participación, Consejería de Salud, Junta de Andalucía [Andalusian Regional Ministry of Health]; 2001. Spanish.

2. World Health Organization. Eliminating measles and rubella and preventing congenital rubella infection: WHO European Region Strategic Plan 2005-2010. Copenhagen: WHO Regional Office for Europe. 2005. Available from: http://www.euro.who. int/InformationSources/Publications/Catalogue/20051123_1

3. Vacunación Infantil, Campaña 2011. [Childhood vaccination, campain 2011]. Sevilla: Consejería de Salud, Junta de Andalucía [Andalusian Regional Ministry of Health]. [Accessed 9 November 2010]. Spanish. Available from: http://www. juntadeandalucia.es/salud/channels/temas/temas_es/P_4 SALUD PUBLICA/P 3 PREVENCION/Vacunas/vacunacion infantil/vacunacion_infantil?perfil=org\&desplegar $=/$ temas_es/P_4_SALUD_PUBLICA/\&idioma=es\&tema=/ temas_es/P_4_SALUD_PUBLICA/P_3_PREVENCION/ Vacunas/vacunacion infantil/\&contenido=/channels/temas/ temas_es/P_4_SALUD_PUBLICA/P_3_PREVENCION/Vacunas/ vacunacion_infantil/vacunacion_infantil 
4. Nieto-Vera J, Masa-Calles J, Dávila J, Molina-Font J, Jiménez M, Gallardo-García V, et al. An outbreak of measles in Algeciras, Spain, 2008 - a preliminary report. Euro Surveill. 2008;13(20):pii=18872. Available from: http://www. eurosurveillance.org/ViewArticle. aspx?Articleld $=18872$

5. Protocolo de Alerta Epidemiológica por Sarampión. [Protocol of an epidemiological alert for measles]. Sevilla: Consejería de Salud, Junta de Andalucía [Andalusian Regional Ministry of Health]; 2008 August 1. Spanish. Available from: http:// www.juntadeandalucia.es/salud/export/sites/csalud/galerias/ documentos/p_4_p_1_vigilancia_de_la_salud/Protocolos_ actuacion/sarampion.pdf

6. Bätzing-Feigenbaum J, Pruckner U, Beyer A, Sinn G, Dinter A, Mankertz A, et al. Spotlight on measles 2010: Preliminary report of an ongoing measles outbreak in a subpopulation with low vaccination coverage in Berlin, Germany, JanuaryMarch 2010. Euro Surveill. 2010;15(13):pii=19527. Available online:http://www.eurosurveillance.org/ViewArticle. aspx?Articleld $=19527$

7. Centro Nacional de Epidemiología. [National Centre of Epidemiology]. Estudio seroepidemiológico: situación de las enfermedades vacunables en España. [Seroepidemiological study: situation of vaccine-preventable diseases in Spain]. Madrid: Instituto de Salud Carlos III; 2000. Spanish. 